\title{
Nursing interventions to promote smoking cessation during pregnancy: An integrative review
}

\author{
Tanya Kintz, Carely Pryor, Holly Shemami, Suha Al-Oballi Kridli \\ School of Nursing, Oakland University, Rochester, USA. \\ Correspondence: Suha Al-Oballi Kridli. Address: School of Nursing, Oakland University, Rochester, USA. \\ Email: kridli@oakland.edu
}

Received: May 8, 2014

Accepted: June 22, 2014

Online Published: July 17, 2014

DOI : $10.5430 /$ jnep.v4n9p77

URL: http://dx.doi.org/10.5430/jnep.v4n9p77

\section{Abstract}

Background: Smoking during their pregnancy or even through a portion of the pregnancy put the unborn child at risk for many complications during pregnancy and after delivery. Teaching smoking cessation is vital to women of childbearing age. Smoking cessation provides immediate and long-term benefits for pregnant women and their children.

Purpose: The purpose of this study is to describe the degree of variability in the methodological approaches and theoretical frameworks of behavioral intervention for smoking cessation during pregnancy.

Methods: The design selected for this research is integrative review. Twenty-four articles were reviewed. The inclusion criteria were: (a) studies published between 2000 and 2013, (b) studies published in the English language, (c) a smoking cessation intervention program that targeted pregnant women, and (d) measurement of smoking status after a smoking cessation intervention was implemented. A data extraction tool developed for the purpose of this study utilizing the frameworks of Cooper (1984), and Stetler and colleagues (1998).

Results: Seventy percent of the reviewed studies reported either smoking cessation or a reduction in smoking as a result of participating in a smoking cessation program. The reviewed studies confirmed that smoking cessation interventions should begin at the beginning of pregnancy, as early pregnancy is a peak opportunity for education. It is highly beneficial if the interventions last throughout the woman's pregnancy to ensure smoking cessation through the duration of the post-partum period.

Conclusion: The majority of the reviewed interventions proved to be highly beneficial with the reduction in smoking or smoking cessation. Additional research is needed to evaluate individual treatment modalities using a side-by-side comparison.

\section{Key words}

Pregnancy, Smoking, Smoking cessation, Nursing interventions, Integrative review

\section{I ntroduction}

"Smoking is the single most important modifiable cause of poor pregnancy outcomes in the US" ${ }^{[1]}$. Smoking during pregnancy has been shown to contribute to adverse outcomes including miscarriage, placental abruption and separation, 
premature rupture of membranes, preterm delivery, low birth weight, increased prenatal mortality, still birth, and sudden infant death syndrome (SIDS) ${ }^{[2]}$.

Women who smoke during their pregnancy or even through a portion of the pregnancy, put their unborn child at risk for many complications during pregnancy and after delivery. Smoking accounts for $10 \%$ of infant mortality, $20 \%-30 \%$ of low birth weight and increased risk for spontaneous abortion and complicated birth ${ }^{[3]}$. Tobacco smoke introduces greater than 4000 potentially teratogenic chemicals into cardiovascular circulation ${ }^{[4]}$. Nicotine from cigarettes has a dose-dependent effect that causes vasoconstriction and decreases the amount of blood and oxygen that reaches the fetus ${ }^{[3]}$. Women who smoke have a $30 \%$ higher chance of delivering prematurely ${ }^{[4]}$. Nicotine readily crosses the placenta and can enter the amniotic fluid, fetal circulation and can be absorbed through the skin of the fetus ${ }^{[4]}$. Long-term nicotine exposure during gestation may reduce nutrient supply and have a direct effect on the cell disposition for genetic instability. This oxidative stress can cause a nicotine induced reactive oxygen species (ROS) which can result in mitochondrial DNA damage making the fetus more prone to have genetic instabilities such as developing lung cancer ${ }^{[4]}$. Studies have shown that between $25 \%$ and $60 \%$ of pregnant smokers quit smoking spontaneously when they learn they are pregnant ${ }^{[2]}$. With this said, teaching smoking cessation is vital to women of childbearing age. Smoking cessation provides immediate and long-term benefits for pregnant women and their young children.

\subsection{Purpose}

The purpose of this integrative review is to describe the degree of variability in the methodological approaches and theoretical frameworks of behavioral intervention for smoking cessation during pregnancy. This review examined the varying smoking cessation interventions employed, the strength of the evidence supporting use of smoking cessation intervention, whether the interventions were guided by the use of a theoretical framework, and to determine whether the intervention successfully demonstrated decreased levels of smoking during pregnancy.

\subsection{Significance to nursing}

According to Vries et al. favorable outcomes have been found, when smoking cessation interventions were implemented by specifically trained health care professionals ${ }^{[6]}$. Care priorities emphasize patient and family education, patient participation in their self-care, promotion of optimal health, provisions of continually competent care, facilitation of entry into the health care system, and the promotion of a safe environment ${ }^{[7]}$. Counseling by a trained provider lasting only 5-15 min is associated with modest but clinically significant effects on cessation rates for pregnant women ${ }^{[3]}$. A recent study by Petersen et al. (2010) showed that interventions for smoking cessation in pregnant women was viewed as an "eye-opener" for questioning normative smoking behaviors in their communities and "door-opener" for enabling behavioral change by increasing faith in the health system and excitement about the pregnancy ${ }^{[8]}$. This integrative review will provide the health care providers with background information about interventions that promote the cessation of smoking during pregnancy.

\subsection{Conceptual framework}

The systematic approach developed by Cooper (1984) was the guiding framework for this integrative review. Cooper identifies the process of conducting an integrative review as encompassing the following five stages: (a) problem formulation, (b) data collection or literature search, (c) data evaluation, (d) data analysis and interpretation, and (e) public presentation of results ${ }^{[9]}$.

The integrative literature review provides several contributions to the scholarly reviewer, which include evaluating the strength of scientific evidence, identifying gaps in past and current research, identifying the need for future research, bridging between related areas of inquiry, identifying central issues in an area, and identifying whether theoretical or conceptual frameworks are utilized ${ }^{[10]}$.

\subsection{Background}

Multiple factors are known to affect whether a pregnant woman will achieve abstinence from smoking during pregnancy or not. Past medical history, demographics, smoking history, smoking status of significant other and other family members 
sharing a living space, quit history, mental health history, perceived self-efficacy, and motivation level of women who smoke during pregnancy all have a role in predicting whether a woman will achieve abstinence ${ }^{[5]}$. Addressing these factors is important in designing an intervention that will be accepted by pregnant women.

Traditional methods to smoking cessation available to non-pregnant smokers are not highly recommended for pregnant smokers. In a study conducted by Gaither, Brunner-Huber, Thompson and Huet-Hudson (2009), nicotine replacement therapy was found to have negative effects on fetuses, and physicians were reluctant to prescribe it to pregnant patients due to the unknown risks to the fetus ${ }^{[11]}$. Gaither et al. (2009) also stated that it is possible for nicotine and other chemicals used in nicotine replacement therapy to build up in the fetus. This is because nicotine is water-soluble; it readily passes through the placental membranes and is not easily metabolized by the fetus. This study concluded that pregnant women who used nicotine replacement therapy had higher rates of low birth weight infants and preterm deliveries ${ }^{[11]}$.

\section{Methods}

The design selected for conducting this research is an integrative review. Integrative reviews are a broad type of research that provides the researcher with experimental and non-experimental research to learn about a certain phenomenon ${ }^{[12]}$. According to Cooper (1982), integrative reviews summarize and synthesize information from various sources that highlight the most relevant issues ${ }^{[13]}$. The inclusion of both experimental and non-experimental research to more fully understand a phenomenon of concern makes the integrative review one of the broadest types of research reviews ${ }^{[12]}$. Integrative literature reviews also contribute to the body of evidence based practice research needed to advance the practice of nursing. "Well-done integrative reviews present the state of the science, contribute to theory development, and have direct applicability to practice and policy" ${ }^{[12]}$.

A data extraction tool developed for the purpose of this study utilizing the frameworks of Cooper (1984), and Stetler and colleagues (1998) was used to ensure accurate and consistent retrieval of data. To ensure rigor, two reviewers tested unitizing reliability ${ }^{[9,14]}$. Intrarater reliability was verified by investigator re-analysis of selected data ${ }^{[15]}$. Developing a clear and concise system for data collection greatly improves the reviewer's capacity to ascertain reliable information from all information sources ${ }^{[10]}$.

Stetler and colleagues (1998) describe six different levels of research. Level I refers to a meta-analysis of multiple controlled studies and is the strongest type of research. Level II illustrates an individual experimental study. Level III indicates a quasi-experimental study, such as nonrandomized controlled single group pre-post test, time series, or matched case-controlled studies. Level IV describes non-experimental study, such as correlational descriptive and qualitative or case studies. Level V indicates a case report or program evaluation data. Lastly, Level VI describes reviewed evidence, based on the opinions of respected authorities. Additionally, "quality from any level can range from A to D and reflects basic scientific credibility of the overall study/project. An A reflects a very well designed study/project. If quality is rated as a $\mathrm{D}$, it is automatically eliminated from consideration" ${ }^{[14]}$. Data extracted from the included studies is presented in Table 1 and consists of: (a) sample characteristics, (b) description of the intervention, (c) outcome measures, (d) measurement points, (e) theoretical framework, (f) results of the study, and (g) level of evidence. The data was extracted from the studies by the authors of this review.

The majority of research articles were obtained by online computer search using the CINAHL and MEDLINE database. The following key words were used: pregnancy, smoking, behavioral modification, interventions, cessation and program. In addition to computer searches, the ancestry approach was utilized to conduct a more extensive literature search. The ancestry approach is the process of gathering information from related publications by reviewing bibliographies of related studies ${ }^{[13]}$. A critical appraisal was conducted to assess the quality of studies that met the inclusion criteria. The criteria used for evaluating methodological quality was based on the (a) level of research significance, (b) characteristics of the study sample, and (c) statistical importance of the results. 


\section{Results}

A total of 24 articles were identified that met the inclusion criteria and addressed smoking intervention programs and implications for health care providers. The research synthesis table is presented in Table 1 . The table is a summary of the reviewed articles with intervention, duration, outcome measures, theoretical framework, and outcomes. Table 2 provides a summary of the types of interventions used or incorporated in this study and their effectiveness.

Table 1. Summary of Reviewed Studies

\begin{tabular}{|c|c|c|c|c|c|c|c|c|}
\hline $\begin{array}{l}\text { Author/ } \\
\text { Year }\end{array}$ & $\begin{array}{l}\text { Sample } \\
\text { Size }\end{array}$ & $\begin{array}{l}\text { Inclusion } \\
\text { Criteria }\end{array}$ & $\begin{array}{l}\text { Description of } \\
\text { Intervention }\end{array}$ & $\begin{array}{l}\text { Measure- } \\
\text { ment }\end{array}$ & $\begin{array}{l}\text { Measure- } \\
\text { ment Points }\end{array}$ & $\begin{array}{l}\text { Theoretical } \\
\text { Framework }\end{array}$ & Outcome & $\begin{array}{l}\text { Level of } \\
\text { Evidence }\end{array}$ \\
\hline $\begin{array}{l}\text { Albrect } \\
\text { et al., } \\
2006\end{array}$ & 142 & $\begin{array}{l}14-19 \text { years old, } \\
12-28 \text { weeks } \\
\text { gestation, } \\
\text { smoker }\end{array}$ & $\begin{array}{l}\text { Teen Fresh Start (TFS)- } \\
\text { didactic content, group } \\
\text { and peer support, 45-60 } \\
\text { minute meetings, and } \\
\text { TFS plus Buddy- a } \\
\text { nonsmoking similar age } \\
\text { friend was present for } \\
\text { intervention meetings }\end{array}$ & $\begin{array}{l}\text { Self report } \\
\text { and saliva } \\
\text { cotinine }\end{array}$ & $\begin{array}{l}\text { Prior to first } \\
\text { intervention, } \\
8 \text { weeks after } \\
\text { randomiza- } \\
\text { tion and } 1 \\
\text { year after } \\
\text { intervention }\end{array}$ & $\begin{array}{l}\text { Cognitive } \\
\text { Behavioral } \\
\text { Theory }\end{array}$ & $\begin{array}{l}\text { a) At baseline, women on } \\
\text { average smoked } 1 / 2 \text { pack per } \\
\text { day. b) } 8 \text { weeks post } \\
\text { randomization, TFS-B group } \\
\text { reported } 40 \% \text { abstinence. } 25 \% \\
\text { were abstinent in the TFS group } \\
\text { and } 15 \% \text { were abstinent in the } \\
\text { usual care group. c) At } 1 \text { year } \\
\text { post intervention, } 10 \% \text { of } \\
\text { TFS-B were abstinent, } 20 \% \text { of } \\
\text { TFS and } 15 \% \text { of control group } \\
\text { were abstinent. }\end{array}$ & II B \\
\hline $\begin{array}{l}\text { Bryce et } \\
\text { al., } 2009\end{array}$ & 65 & $\begin{array}{l}25 \text { years or } \\
\text { younger, } \\
\text { smoker, no } \\
\text { gestational } \\
\text { inclusion } \\
\text { criteria }\end{array}$ & $\begin{array}{l}\text { Initial telephone contact } \\
\text { and subsequent } \\
\text { meetings with women } \\
\text { and partners or a friend } \\
\text { using motivational } \\
\text { interviewing at } \\
\text { baseline, } 3 \text { months and } \\
12 \text { months. Nicotine } \\
\text { replacement therapy } \\
\text { was dispensed by a } \\
\text { midwife. }\end{array}$ & $\begin{array}{l}\text { Self report } \\
\text { and } \\
\text { expired } \\
\text { carbon } \\
\text { monoxide }\end{array}$ & $\begin{array}{l}3 \text { months and } \\
12 \text { months } \\
\text { post delivery. }\end{array}$ & $\begin{array}{l}\text { None } \\
\text { identified }\end{array}$ & $\begin{array}{l}\text { a) At baseline, } 39 \% \text { were } \\
\text { current smokers. b) At } 3 \\
\text { months, } 30 \% \text { of smokers had } \\
\text { decreased amount of cigarettes } \\
\text { smoked, almost } 4 \% \text { had } \\
\text { increased the amount of } \\
\text { cigarettes smoked per day and } \\
17 \% \text { had no change. c) At } 12 \\
\text { months, } 6 \% \text { had quit smoking, } \\
10 \% \text { decreased the number of } \\
\text { daily cigarettes and } 14 \% \text { had no } \\
\text { change. No one had an increase } \\
\text { in number of cigarettes smoked } \\
\text { per day. }\end{array}$ & III B \\
\hline $\begin{array}{l}\text { Bullock } \\
\text { et al., } \\
2009\end{array}$ & 695 & $\begin{array}{l}18 \text { years or } \\
\text { older, reported } \\
\text { smoking at least } \\
1 \text { cigarette per } \\
\text { day, english } \\
\text { speaking, and } \\
\text { less than } 24 \\
\text { weeks } \\
\text { gestation. }\end{array}$ & $\begin{array}{l}\text { Weekly telephone call, } \\
24 \text { hour a day access to } \\
\text { a nurse for support. } 1 \\
\text { booklet was given to } \\
\text { women at clinic visit } \\
\text { and remaining } 7 \text { were } \\
\text { mailed. } 3 \text { separate } \\
\text { intervention groups } \\
\text { tested, one received the } \\
\text { books plus nurse } \\
\text { support, one with nurse } \\
\text { support alone and one } \\
\text { with booklets alone. }\end{array}$ & $\begin{array}{l}\text { Self report } \\
\text { and saliva } \\
\text { cotinine }\end{array}$ & $\begin{array}{l}\text { Monthly } \\
\text { salivary } \\
\text { samples were } \\
\text { collected }\end{array}$ & $\begin{array}{l}\text { None } \\
\text { identified }\end{array}$ & $\begin{array}{l}\text { a) At delivery, no statistically } \\
\text { significant differences were } \\
\text { present between the } \\
\text { intervention groups and the } \\
\text { control groups. The nurse } \\
\text { support only group had } \\
\text { marginally higher abstinence } \\
\text { rates at } 22 \% \text { compared to } 17 \% \\
\text { in the intervention group. b) } \\
\text { Post delivery, abstinence rates } \\
\text { dropped by an average of } \\
12.65 \% \text {. }\end{array}$ & II C \\
\hline $\begin{array}{l}\text { Campbe } \\
\text { ll, et al., } \\
2006\end{array}$ & 5,145 & $\begin{array}{l}16 \text { years or } \\
\text { older, smoker, } \\
\text { generally } \\
\text { healthy, English } \\
\text { speaking }\end{array}$ & $\begin{array}{l}2 \text { experimental groups: } \\
1 \text { received a single } \\
\text { mailing that had } \\
\text { information on the } \\
\text { benefits of quitting } \\
\text { smoking and available } \\
\text { at clinics were videos, } \\
\text { charts and self help kits. } \\
\text { The second group } \\
\text { received written } \\
\text { information, resources, } \\
\text { and computerized } \\
\text { activities for women to } \\
\text { report their smoking } \\
\text { cessation activities. }\end{array}$ & $\begin{array}{l}\text { Self report } \\
\text { and } \\
\text { expired } \\
\text { carbon } \\
\text { monoxide }\end{array}$ & $\begin{array}{l}\text { Once at study } \\
\text { entrance and } \\
\text { once at a } \\
\text { subsequent } \\
\text { clinic visit }\end{array}$ & $\begin{array}{l}\text { Roger's } \\
\text { Model }\end{array}$ & $\begin{array}{l}\text { Neither of the intervention } \\
\text { groups had statistically } \\
\text { significant smoking cessation } \\
\text { rates. }\end{array}$ & II C \\
\hline $\begin{array}{l}\text { Cope et } \\
\text { al., } 2003\end{array}$ & 192 & $\begin{array}{l}\text { Pregnant } \\
\text { smokers }\end{array}$ & $\begin{array}{l}\text { Women set quit dates } \\
\text { and were given written } \\
\text { material on how to quit. } \\
\text { Clinic staff were } \\
\text { encouraging and } \\
\text { supportive, offered } \\
\text { feedback and } \\
\text { empowerment. }\end{array}$ & $\begin{array}{l}\text { Self report } \\
\text { and urine } \\
\text { cotinine }\end{array}$ & $\begin{array}{l}\text { Measurement } \\
\text { on each clinic } \\
\text { visit up to } \\
\text { and including } \\
\text { the } 36 \text { th week } \\
\text { clinic visit. }\end{array}$ & $\begin{array}{l}\text { None } \\
\text { identified }\end{array}$ & $\begin{array}{l}\text { a) The rate of smoking } \\
\text { cessation in the intervention } \\
\text { group was } 16.2 \% \text {, and } 8 \% \text { in } \\
\text { the control. b) } 33 \% \\
\text { significantly decreased their } \\
\text { cigarette use in the intervention } \\
\text { group, and } 23 \% \text { reduced } \\
\text { smoking in the control group. }\end{array}$ & II B \\
\hline
\end{tabular}


Table 1. (continued.)

\begin{tabular}{|c|c|c|c|c|c|c|c|c|}
\hline $\begin{array}{l}\text { Author/ } \\
\text { Year }\end{array}$ & $\begin{array}{l}\text { Sample } \\
\text { Size }\end{array}$ & $\begin{array}{l}\text { Inclusion } \\
\text { Criteria }\end{array}$ & $\begin{array}{l}\text { Description of } \\
\text { Intervention }\end{array}$ & $\begin{array}{l}\text { Measure- } \\
\text { ment }\end{array}$ & $\begin{array}{l}\text { Measure- } \\
\text { ment Points }\end{array}$ & $\begin{array}{l}\text { Theoretical } \\
\text { Framework }\end{array}$ & Outcome & $\begin{array}{l}\text { Level of } \\
\text { Evidence }\end{array}$ \\
\hline $\begin{array}{l}\text { Dornelas } \\
\text { et al., } \\
2006\end{array}$ & 105 & $\begin{array}{l}\text { Pregnant } \\
\text { women } 18 \text { years } \\
\text { or older, } \\
\text { smokers, } 30 \\
\text { weeks or less } \\
\text { gestation, with } \\
\text { no other } \\
\text { chemical } \\
\text { dependence, no } \\
\text { psychiatric } \\
\text { illness and } \\
\text { access to a } \\
\text { telephone }\end{array}$ & $\begin{array}{l}90 \text { minute } \\
\text { psychotherapy session } \\
\text { and bimonthly } \\
\text { telephone calls from the } \\
\text { therapist, and monthly } \\
\text { calls after delivery }\end{array}$ & $\begin{array}{l}\text { Self report } \\
\text { and } \\
\text { expired } \\
\text { carbon } \\
\text { monoxide }\end{array}$ & $\begin{array}{l}\text { Measurement } \\
\text { by self report } \\
\text { was during } \\
\text { each } \\
\text { telephone call, } \\
\text { and expired } \\
\text { carbon } \\
\text { monoxide was } \\
\text { collected at } \\
\text { the end of } \\
\text { pregnancy } \\
\text { and } 6 \text { months } \\
\text { post partum }\end{array}$ & $\begin{array}{l}\text { None } \\
\text { identified }\end{array}$ & $\begin{array}{l}\text { a) At the end of pregnancy, } \\
\text { smoking abstinence rates } \\
\text { were } 28.3 \% \text { in the } \\
\text { intervention group and } 9.6 \% \\
\text { in the control group. b) At } 6 \\
\text { months post partum, } \\
\text { abstinence rates decreased } \\
\text { to } 9.4 \% \text { in the intervention } \\
\text { group and 3.8\% in the } \\
\text { control group. }\end{array}$ & II B \\
\hline $\begin{array}{l}\text { Edwards } \\
\text { et al., } \\
2008\end{array}$ & 12,133 & $\begin{array}{l}\text { Pregnant } \\
\text { smokers } \\
\text { receiving WIC } \\
\text { benefits }\end{array}$ & $\begin{array}{l}\text { Individual counseling } \\
\text { and treatment plans } \\
\text { with educational } \\
\text { information and written } \\
\text { material, intervention } \\
\text { utilized "5 A's" } \\
\text { guideline }\end{array}$ & Self report & $\begin{array}{l}\text { Once at the } \\
\text { beginning of } \\
\text { the study and } \\
\text { once after } \\
\text { delivery }\end{array}$ & $\begin{array}{l}\text { None } \\
\text { identified }\end{array}$ & $\begin{array}{l}\text { a) } 24.2 \% \text { of participants that } \\
\text { received counseling and } \\
\text { used the self help guide quit } \\
\text { smoking, } 20.9 \% \text { of } \\
\text { participants that used the } \\
\text { self help guide but did not } \\
\text { receive counseling quit } \\
\text { smoking. b) the earlier in } \\
\text { pregnancy the women } \\
\text { sought prenatal care and } \\
\text { entered this study, the more } \\
\text { likely they were to quit } \\
\text { smoking. }\end{array}$ & III B \\
\hline $\begin{array}{l}\text { Ferreira- } \\
\text { Borges, } \\
2005\end{array}$ & 57 & $\begin{array}{l}\text { Pregnant } \\
\text { smokers less } \\
\text { than } 28 \text { weeks } \\
\text { gestation }\end{array}$ & $\begin{array}{l}1 \text { motivational } \\
\text { interviewing session } \\
\text { and written materials }\end{array}$ & $\begin{array}{l}\text { Self report } \\
\text { and } \\
\text { expired } \\
\text { carbon } \\
\text { monoxide }\end{array}$ & $\begin{array}{l}\text { Measurement } \\
\text { points at first } \\
\text { visit and } 2 \\
\text { month follow } \\
\text { up }\end{array}$ & $\begin{array}{l}\text { None } \\
\text { identified }\end{array}$ & $\begin{array}{l}\text { a) After intervention, } 33 \% \\
\text { of experimental group had } \\
\text { achieved abstinence and } \\
\text { only } 8 \% \text { of control group } \\
\text { was abstinent. b) } 66 \% \text { of } \\
\text { experimental group reduced } \\
\text { smoking status to less than } 5 \\
\text { per day. c) The mean } \\
\text { amount of cigarettes } \\
\text { smoked per day decreased } \\
\text { by } 51 \% \text { in the experimental } \\
\text { group and by } 19.7 \% \text { in the } \\
\text { control group. }\end{array}$ & III B \\
\hline $\begin{array}{l}\text { Heil et } \\
\text { al., } 2008\end{array}$ & 82 & $\begin{array}{l}\text { Pregnant } \\
\text { smokers } 20 \text { or } \\
\text { less weeks of } \\
\text { gestation }\end{array}$ & $\begin{array}{l}\text { Women set quit dates, } \\
\text { and were given } \\
\text { vouchers with monetary } \\
\text { value for cessation } \\
\text { efforts based on } \\
\text { negative nicotine tests. } \\
\text { With each subsequent } \\
\text { negative test, monetary } \\
\text { amount of vouchers } \\
\text { increased. If nicotine } \\
\text { test was positive, no } \\
\text { voucher was given. } \\
\text { This continued until the } \\
\text { women delivered. }\end{array}$ & $\begin{array}{l}\text { Self report, } \\
\text { urine and } \\
\text { expired } \\
\text { carbon } \\
\text { monoxide. }\end{array}$ & $\begin{array}{l}\text { Measurement } \\
\text { once a week } \\
\text { for } 4 \text { weeks, } \\
\text { every other } \\
\text { week for } 8 \\
\text { weeks and } \\
\text { once more at } \\
24 \text { weeks. }\end{array}$ & $\begin{array}{l}\text { None } \\
\text { identified }\end{array}$ & $\begin{array}{l}\text { a) Abstinence levels at end } \\
\text { of pregnancy and } 12 \text { weeks } \\
\text { post partum were higher in } \\
\text { group receiving vouchers } \\
\text { contingent on nicotine } \\
\text { levels than group receiving } \\
\text { vouchers independent of } \\
\text { smoking status. b) At } 3 \\
\text { months post partum, } \\
\text { abstinence rates were only } \\
\text { 5\% greater in the group } \\
\text { receiving vouchers } \\
\text { dependant on smoking } \\
\text { status. c) No data was } \\
\text { available to signify a } \\
\text { decrease in amount of } \\
\text { cigarettes smoked. }\end{array}$ & II B \\
\hline $\begin{array}{l}\text { Hennrik } \\
\text { us et al., } \\
2010\end{array}$ & 82 & $\begin{array}{l}\text { At least } 18 \text { years } \\
\text { old, smoker, } 1 \text { st } \\
\text { or } 2 \text { nd trimester }\end{array}$ & $\begin{array}{l}\text { Women identified a } \\
\text { support person and they } \\
\text { made a scrapbook of the } \\
\text { pregnancy together, but } \\
\text { attended smoking } \\
\text { cessation meetings } \\
\text { separately. Monthly } \\
\text { phone calls were made } \\
\text { by midwives to women } \\
\text { and support persons. }\end{array}$ & $\begin{array}{l}\text { Self report } \\
\text { only }\end{array}$ & $\begin{array}{l}\text { Measurement } \\
\text { took place at } \\
\text { baseline, once } \\
\text { prior to } \\
\text { delivery date } \\
\text { and three } \\
\text { months post } \\
\text { partum. }\end{array}$ & $\begin{array}{l}\text { None } \\
\text { identified }\end{array}$ & $\begin{array}{l}\text { A) } 13 \% \text { of participants in } \\
\text { experimental group had quit } \\
\text { smoking, compared to } 3.6 \% \\
\text { in the control group. b) } 9.3 \% \\
\text { of participants in } \\
\text { experimental group were } \\
\text { still abstinent at three } \\
\text { months post partum and } \\
\text { none of the control group } \\
\text { were abstinent. c) } \\
\text { participants who chose } \\
\text { friends as opposed to family } \\
\text { members were } 15 \% \text { more } \\
\text { likely to quit smoking. }\end{array}$ & II B \\
\hline
\end{tabular}

(Table 1 continued on page 82) 
Table 1. (continued.)

\begin{tabular}{|c|c|c|c|c|c|c|c|c|}
\hline $\begin{array}{l}\text { Author/ } \\
\text { Year } \\
\end{array}$ & $\begin{array}{l}\text { Sample } \\
\text { Size } \\
\end{array}$ & $\begin{array}{l}\text { Inclusion } \\
\text { Criteria } \\
\end{array}$ & $\begin{array}{l}\text { Description of } \\
\text { Intervention }\end{array}$ & $\begin{array}{l}\text { Measure- } \\
\text { ment }\end{array}$ & $\begin{array}{l}\text { Measure- } \\
\text { ment Points }\end{array}$ & $\begin{array}{l}\text { Theoretical } \\
\text { Framework }\end{array}$ & Outcome & $\begin{array}{l}\text { Level of } \\
\text { Evidence }\end{array}$ \\
\hline $\begin{array}{l}\text { Jaakkola } \\
\text { et al., } \\
2001\end{array}$ & 458 & $\begin{array}{l}\text { Pregnant } \\
\text { smokers }\end{array}$ & $\begin{array}{l}\text { Women received health } \\
\text { education material, } \\
\text { smoking cessation } \\
\text { material, and were visited } \\
\text { at home, husbands } \\
\text { received smoking } \\
\text { cessation information and } \\
\text { maternity training. }\end{array}$ & $\begin{array}{l}\text { Self report } \\
\text { and hair } \\
\text { nicotine } \\
\text { concentrati } \\
\text { on }\end{array}$ & $\begin{array}{l}\text { Hair sample } \\
\text { was collected } \\
\text { once at the } \\
\text { end of the } \\
\text { study, self } \\
\text { report was } \\
\text { collected } \\
\text { once at the } \\
\text { beginning of } \\
\text { the study and } \\
\text { once at the } \\
\text { end. }\end{array}$ & $\begin{array}{l}\text { None } \\
\text { identified }\end{array}$ & $\begin{array}{l}\text { a) Biochemically validated quit } \\
\text { rates for the intervention group } \\
\text { were } 13.4 \% \text { and } 9.2 \% \text { in the } \\
\text { control group. b) the } \\
\text { intervention group reduced } \\
\text { cigarette consumption by an } \\
\text { average of } 1.41 \text { cigarettes per } \\
\text { day, and the control group } \\
\text { reduced cigarette consumption } \\
\text { by } 1.28 \text { cigarettes per day. }\end{array}$ & III B \\
\hline $\begin{array}{l}\text { Kataray } \\
\text { et al., } \\
2009\end{array}$ & 38 & $\begin{array}{l}\text { Pregnant } \\
\text { smokers } \\
\text { less than } \\
16 \text { weeks } \\
\text { gestation }\end{array}$ & $\begin{array}{l}\text { Motivational interviews } \\
\text { were conducted, written } \\
\text { materials were distributed, } \\
\text { and women set quit dates. } \\
8 \text { home visits were made } \\
\text { by nurses, } 5 \text { were focused } \\
\text { on the intervention and } 3 \\
\text { were for follow up } \\
\text { purposes. }\end{array}$ & $\begin{array}{l}\text { Self report, } \\
\text { urine } \\
\text { cotinine } \\
\text { and } \\
\text { expired } \\
\text { carbon } \\
\text { monoxide. }\end{array}$ & $\begin{array}{l}\text { Measurement } \\
\text { points at each } \\
\text { home visit. }\end{array}$ & $\begin{array}{l}\text { Transtheoreti } \\
\text { cal model of } \\
\text { change }\end{array}$ & $\begin{array}{l}\text { a) At the end of the } \\
\text { intervention, } 39.5 \% \text { of women } \\
\text { had stopped smoking. b) } 44.7 \% \\
\text { of women had reduced smoking } \\
\text { by } 60 \% \text { from starting rate. c) } \\
\text { self efficacy scores had } \\
\text { increased by } 27 \% \text { after the } \\
\text { intervention. }\end{array}$ & III B \\
\hline $\begin{array}{l}\text { Lando et } \\
\text { al., } 2001\end{array}$ & 4,213 & $\begin{array}{l}\text { Pregnant } \\
\text { women } \\
\text { who } \\
\text { currently } \\
\text { smoked or } \\
\text { quit } \\
\text { recently }\end{array}$ & $\begin{array}{l}\text { The HOPP intervention } \\
\text { consisted of telephone } \\
\text { counseling, written } \\
\text { materials on quitting } \\
\text { smoking and relapse } \\
\text { prevention materials. The } \\
\text { STORK intervention was } \\
\text { individualized and used } \\
\text { brief motivational } \\
\text { interviewing and support } \\
\text { based on the woman's } \\
\text { readiness to quit stage }\end{array}$ & $\begin{array}{l}\text { Self report } \\
\text { and Saliva } \\
\text { cotinine } \\
\text { for the } \\
\text { HOPP } \\
\text { interventio } \\
n\end{array}$ & $\begin{array}{l}\text { Measurement } \\
\text { at } 8 \text { weeks } \\
\text { post partum, } \\
6 \text { months post } \\
\text { partum and } \\
12 \text { months } \\
\text { post partum }\end{array}$ & $\begin{array}{l}\text { Transtheoreti } \\
\text { cal model of } \\
\text { change }\end{array}$ & $\begin{array}{l}\text { a) The results of the HOPP } \\
\text { intervention did not indicate } \\
\text { any smoking cessation statistics } \\
\text { during pregnancy, but reported } \\
\text { that relapse rates had } \\
\text { decreased. b) the STORK } \\
\text { intevention achieved } 39 \% \\
\text { abstinence in the intervention } \\
\text { group and } 29 \% \text { in the control } \\
\text { group, but no differences in } \\
\text { quit rates were noted between } \\
\text { the groups at } 12 \text { months post } \\
\text { partum }\end{array}$ & II B \\
\hline $\begin{array}{l}\text { Lawrenc } \\
\text { e et al., } \\
2003\end{array}$ & 918 & $\begin{array}{l}\text { Pregnant } \\
\text { smokers } \\
16 \text { years or } \\
\text { older }\end{array}$ & $\begin{array}{l}\text { Six } 30 \text { page self help } \\
\text { manuals were distributed, } \\
\text { one for each stage of } \\
\text { change. Three } 15 \text { minute } \\
\text { meetings were held to } \\
\text { discuss smoking } \\
\text { cessation. One group was } \\
\text { given a computer program } \\
\text { in addition to the self help } \\
\text { manuals and the meetings. }\end{array}$ & $\begin{array}{l}\text { Self report } \\
\text { and urine } \\
\text { cotinine }\end{array}$ & $\begin{array}{l}\text { Measurement } \\
\text { points were at } \\
30 \text { weeks } \\
\text { gestation and } \\
10 \text { days post } \\
\text { delivery. }\end{array}$ & $\begin{array}{l}\text { Trans-theoret } \\
\text { ical model of } \\
\text { change }\end{array}$ & $\begin{array}{l}\text { a) Intervention was not } \\
\text { statistically shown to improve } \\
\text { smoking quit rates. b) there } \\
\text { were very little differences in } \\
\text { the smoking rates of the } \\
\text { experimental and control } \\
\text { groups, only } 3 \% \text { of the } \\
\text { intervention group achieved } \\
\text { cessation. }\end{array}$ & II C \\
\hline $\begin{array}{l}\text { Malchod } \\
\text { i et al., } \\
2003\end{array}$ & 142 & $\begin{array}{l}\text { Pregnant } \\
\text { smokers } \\
18 \text { years or } \\
\text { older and } \\
\text { less than } \\
20 \text { weeks } \\
\text { gestation }\end{array}$ & $\begin{array}{l}\text { Peer counseling either by } \\
\text { telephone call, home visit } \\
\text { or clinic meeting was used } \\
\text { from community health } \\
\text { outreach workers in } \\
\text { addition to usual care. } \\
\text { Role playing and } \\
\text { motivational interviewing } \\
\text { were used in } 2 \text { meetings } \\
\text { totalling } 5 \text { hours in } \\
\text { duration. }\end{array}$ & $\begin{array}{l}\text { Self report, } \\
\text { expired } \\
\text { carbon } \\
\text { monoxide } \\
\text { and urine } \\
\text { cotinine }\end{array}$ & $\begin{array}{l}\text { Measurement } \\
\text { was at } \\
\text { baseline and } \\
\text { at } 36 \text { weeks } \\
\text { gestation. }\end{array}$ & $\begin{array}{l}\text { None } \\
\text { identified }\end{array}$ & $\begin{array}{l}\text { a) Intervention was more } \\
\text { successful at reducing smoking } \\
\text { than achieving abstinence. } 24 \% \\
\text { of women in experimental } \\
\text { group were abstinent, and } 20 \% \\
\text { in control group were abstinent. } \\
\text { b) peer counseling had greatest } \\
\text { effect on reducing amount of } \\
\text { cigarettes per day on those who } \\
\text { smoked } 10 \text { or more per day at } \\
\text { the beginning of the study. }\end{array}$ & II B \\
\hline $\begin{array}{l}\text { McGow } \\
\text { an et al., } \\
2010\end{array}$ & 1,936 & $\begin{array}{l}\text { Pregnant } \\
\text { smokers }\end{array}$ & $\begin{array}{l}\text { Three phone calls and one } \\
\text { clinic visit were used, } \\
\text { based on motivational } \\
\text { interviewing. Women } \\
\text { were sent text messages } \\
\text { by nurses. Nicotine } \\
\text { replacement therapy was } \\
\text { dispensed by a } \\
\text { pharmacist. }\end{array}$ & $\begin{array}{l}\text { Self report } \\
\text { and } \\
\text { expired } \\
\text { carbon } \\
\text { monoxide }\end{array}$ & $\begin{array}{l}\text { Measurement } \\
\text { points were } \\
\text { weekly for } 7 \\
\text { weeks }\end{array}$ & $\begin{array}{l}\text { None } \\
\text { identified }\end{array}$ & $\begin{array}{l}\text { a) } 32 \% \text { of smokers achieved } \\
\text { cessation b) no data available } \\
\text { on long term quit rates }\end{array}$ & II B \\
\hline $\begin{array}{l}\text { McLeod } \\
\text { et al., } \\
2003\end{array}$ & 297 & $\begin{array}{l}\text { Pregnant } \\
\text { smokers }\end{array}$ & $\begin{array}{l}\text { Brief motivational } \\
\text { interviewing for smoking } \\
\text { cessation, women in } \\
\text { separate group received } \\
\text { intervention to increase } \\
\text { breast feeding, and third } \\
\text { group received both } \\
\text { smoking cessation and } \\
\text { breast feeding } \\
\text { intervention }\end{array}$ & $\begin{array}{l}\text { Self report } \\
\text { and serum } \\
\text { cotinine }\end{array}$ & $\begin{array}{l}\text { Measurement } \\
\text { points at } \\
\text { beginning of } \\
\text { study, } 28 \\
\text { weeks } \\
\text { gestation, six } \\
\text { weeks post } \\
\text { partum and } \\
\text { four months } \\
\text { post partum }\end{array}$ & $\begin{array}{l}\text { None } \\
\text { identified }\end{array}$ & $\begin{array}{l}\text { a) Women receiving the } \\
\text { smoking cessation only } \\
\text { intervention had abstinence } \\
\text { rates of } 22 \% \text { at } 28 \text { weeks } \\
\text { gestation and } 9 \% \text { in the control } \\
\text { group; } 17 \% \text { at } 4 \text { months post } \\
\text { delivery with } 13 \% \text { in the } \\
\text { control group. b) women who } \\
\text { received both the smoking } \\
\text { cessation intervention and the } \\
\text { breast feeding intervention had } \\
\text { lower smoking abstinence rates } \\
\text { at all but } 1 \text { data collection point. }\end{array}$ & II B \\
\hline
\end{tabular}


Table 1. (continued.)

\begin{tabular}{|c|c|c|c|c|c|c|c|c|}
\hline $\begin{array}{l}\text { Author/ } \\
\text { Year } \\
\end{array}$ & $\begin{array}{l}\text { Sample } \\
\text { Size }\end{array}$ & $\begin{array}{l}\text { Inclusion } \\
\text { Criteria } \\
\end{array}$ & $\begin{array}{l}\text { Description of } \\
\text { Intervention } \\
\end{array}$ & $\begin{array}{l}\text { Measure- } \\
\text { ment }\end{array}$ & $\begin{array}{l}\text { Measure- } \\
\text { ment Points }\end{array}$ & $\begin{array}{l}\text { Theoretical } \\
\text { Framework }\end{array}$ & Outcome & $\begin{array}{l}\text { Level of } \\
\text { Evidence }\end{array}$ \\
\hline $\begin{array}{l}\text { Moore et } \\
\text { al., } 2002\end{array}$ & 1,527 & $\begin{array}{l}\text { Pregnant } \\
\text { smokers age } \\
16 \text { or older, } \\
\text { less than } 17 \\
\text { weeks } \\
\text { gestation and } \\
\text { able to speak } \\
\text { english. }\end{array}$ & $\begin{array}{l}\text { Five self help } \\
\text { booklets were } \\
\text { distributed, the first } \\
\text { one was given at an } \\
\text { office visit and the } \\
\text { 2nd-5th one were } \\
\text { mailed to } \\
\text { participants. }\end{array}$ & $\begin{array}{l}\text { Self report } \\
\text { and urine } \\
\text { cotinine }\end{array}$ & $\begin{array}{l}\text { Measurement } \\
\text { was once at the } \\
\text { beginning of the } \\
\text { study and again } \\
\text { at } 26 \text { weeks } \\
\text { gestation }\end{array}$ & $\begin{array}{l}\text { None } \\
\text { identified }\end{array}$ & $\begin{array}{l}\text { a) Intervention was ineffective; } \\
18.8 \% \text { of experimental group } \\
\text { achieved cessation compared to } \\
20.7 \% \text { in control group. b) self } \\
\text { reported quit rates were higher } \\
\text { than biochemically validated } \\
\text { quit rates. }\end{array}$ & II C \\
\hline $\begin{array}{l}\text { Ondersm } \\
\text { a, et al. } \\
2011\end{array}$ & 110 & $\begin{array}{l}18 \text { years or } \\
\text { older, being } \\
\text { no further } \\
\text { than } 27 \\
\text { weeks into } \\
\text { gestation }\end{array}$ & $\begin{array}{l}\text { (a) a } \\
\text { computer-delivered } \\
\text { 5As-based brief } \\
\text { intervention } \\
\text { (CD-5As) and (b) a } \\
\text { computer-assisted, } \\
\text { simplified, and } \\
\text { low-intensity } \\
\text { contingency } \\
\text { management } \\
\text { (CM-Lite). }\end{array}$ & $\begin{array}{l}\text { Self-report } \\
\text { of } \\
\text { smoking, } \\
\text { urine } \\
\text { cotinine, } \\
\text { and breath } \\
\text { CO were } \\
\text { measured }\end{array}$ & $\begin{array}{l}10 \text { weeks } \\
\text { following } \\
\text { randomization. }\end{array}$ & $\begin{array}{l}\text { None } \\
\text { identified }\end{array}$ & $\begin{array}{l}\text { CM-Lite, did not appear to } \\
\text { facilitate abstinence in this } \\
\text { sample. However, our findings } \\
\text { suggest that the brief } \\
\text { computer-delivered } \\
\text { motivational intervention } \\
\text { (CD-5As) was well accepted by } \\
\text { participants, was associated } \\
\text { with increases in state } \\
\text { motivation, and showed } \\
\text { promising results in terms of } \\
\text { abstinence and help-seeking. }\end{array}$ & IB \\
\hline $\begin{array}{l}\text { Oien et } \\
\text { al., } 2008\end{array}$ & 2,132 & $\begin{array}{l}\text { Pregnant } \\
\text { women who } \\
\text { spoke } \\
\text { Norwegian }\end{array}$ & $\begin{array}{l}\text { Brief smoking } \\
\text { cessation counseling } \\
\text { at each clinic visit, } \\
\text { women whose } \\
\text { significant other } \\
\text { smoked were } \\
\text { encouraged to bring } \\
\text { him }\end{array}$ & Self report & $\begin{array}{l}\text { Measurement at } \\
\text { beginning of } \\
\text { study and once } \\
\text { at the last clinic } \\
\text { visit }\end{array}$ & $\begin{array}{l}\text { None } \\
\text { identified }\end{array}$ & $\begin{array}{l}\text { a) Intervention was ineffective } \\
\text { at achieving smoking cessation, } \\
\text { little differences existed } \\
\text { between experimental and } \\
\text { control group. Data showed } \\
\text { that most of the women who } \\
\text { quit smoking during pregnancy } \\
\text { quit before inclusion in this } \\
\text { study. }\end{array}$ & III C \\
\hline $\begin{array}{l}\text { Patten et } \\
\text { al., } 2010\end{array}$ & 35 & $\begin{array}{l}\text { Pregnant } \\
\text { smokers at } \\
\text { least } 18 \text { years } \\
\text { old, } 24 \text { weeks } \\
\text { or less } \\
\text { gestation, } \\
\text { planning to } \\
\text { quit smoking } \\
\text { in the next } 30 \\
\text { days that had } \\
\text { access to a } \\
\text { telephone and } \\
\text { DVD player }\end{array}$ & $\begin{array}{l}\text { Educational } \\
\text { intervention included } \\
\text { a video, cessation } \\
\text { guide, and telephone } \\
\text { counseling }\end{array}$ & $\begin{array}{l}\text { Self report } \\
\text { and saliva } \\
\text { cotinine }\end{array}$ & $\begin{array}{l}\text { Measurement at } \\
\text { beginning of } \\
\text { study and once } \\
\text { at study follow } \\
\text { up }\end{array}$ & $\begin{array}{l}\text { Social } \\
\text { cognitive } \\
\text { theory }\end{array}$ & $\begin{array}{l}\text { a) This intervention was not } \\
\text { effective, } 6 \% \text { of the control } \\
\text { group had biochemically } \\
\text { confirmed smoking abstinence, } \\
\text { and } 0 \% \text { of the intervention } \\
\text { group was abstinent. b) The } \\
\text { low rate of enrollment in this } \\
\text { study indicates that it is not } \\
\text { feasible or acceptable }\end{array}$ & II C \\
\hline $\begin{array}{l}\text { Peden et } \\
\text { al., } 2008\end{array}$ & 16 & $\begin{array}{l}\text { Pregnant } \\
\text { smokers } 18 \\
\text { years or } \\
\text { older, } 25 \\
\text { weeks or less } \\
\text { gestation }\end{array}$ & $\begin{array}{l}\text { Four 90-minute } \\
\text { group sessions } \\
\text { focused on negative } \\
\text { thinking and } \\
\text { depressive behaviors } \\
\text { during pregnancy. } \\
\text { Self help at home } \\
\text { sessions included } \\
\text { audio tapes and } \\
\text { positive affirmations. }\end{array}$ & $\begin{array}{l}\text { Self report } \\
\text { and urine } \\
\text { cotinine }\end{array}$ & $\begin{array}{l}\text { Measurement } \\
\text { was once at } \\
\text { baseline and } \\
\text { once after } \\
\text { intervention } \\
\text { was completed. }\end{array}$ & $\begin{array}{l}\text { Cognitive } \\
\text { Behavioral } \\
\text { Theory }\end{array}$ & $\begin{array}{l}\text { a) The experimental group } \\
\text { reported less depressive } \\
\text { symptoms and hopelessness } \\
\text { than control group. b) self } \\
\text { reported cigarette use did not } \\
\text { change significantly, however } \\
\text { the mean urine cotinine test } \\
\text { increased by } 1.1 \text { on the } \\
\text { Accutest Vicometer. }\end{array}$ & III C \\
\hline $\begin{array}{l}\text { Vries et } \\
\text { al., } 2006\end{array}$ & 318 & $\begin{array}{l}\text { Pregnant } \\
\text { smokers who } \\
\text { had not been } \\
\text { pregnant } \\
\text { more than } \\
\text { twice and } \\
\text { spoke dutch }\end{array}$ & $\begin{array}{l}\text { Intervention } \\
\text { consisted of a video, } \\
\text { self-help manual, a } \\
\text { booklet for the } \\
\text { woman's partner, and } \\
\text { health counseling by } \\
\text { midwives }\end{array}$ & $\begin{array}{l}\text { Self report } \\
\text { and urine } \\
\text { cotinine }\end{array}$ & $\begin{array}{l}\text { Measurement at } \\
\text { baseline, } 6 \\
\text { weeks after the } \\
\text { intervention and } \\
6 \text { weeks post } \\
\text { partum }\end{array}$ & $\begin{array}{l}\text { Theory of } \\
\text { planned } \\
\text { behavior, } \\
\text { social } \\
\text { cognitive } \\
\text { theory, and } \\
\text { the trans- } \\
\text { theoretical } \\
\text { model of } \\
\text { change }\end{array}$ & $\begin{array}{l}\text { a) The experimental group had } \\
19 \% \text { abstinence at } 6 \text { weeks after } \\
\text { the intervention, the control } \\
\text { group had } 7 \% \text { abstinence. At } 6 \\
\text { weeks post partum the } \\
\text { intervention group reported } \\
12 \% \text { abstinence, and the control } \\
\text { group reported } 3 \% \text { abstinence. } \\
\text { b) the partner intervention was } \\
\text { unsuccessful at decreasing } \\
\text { smoking rates }\end{array}$ & II B \\
\hline
\end{tabular}

\subsection{Theory framework}

Only eight of the twenty-four studies applied theoretical frameworks. Two studies reported the use of the transtheoretical model of change consisting of Kataray et al., (2009) and Lawrence et al. (2003) ${ }^{[16,17]}$. Transtheoretical model of change was developed in 1982 and was based on social learning theories. The main concept of the transtheoretical model of change is staging behavior change. The five stages in this model are: precontemplation, contemplation, preparation, action, and maintenance. The transtheoretical model of change is highly applicable to smoking cessation and is the basis of many smoking cessation studies ${ }^{[16]}$. 
Table 2. Interventions Used in Reviewed Studies

\begin{tabular}{|c|c|c|c|c|c|c|c|c|c|c|}
\hline $\begin{array}{l}\text { Interventions } \\
\text { utilized in smoking } \\
\text { cessation programs }\end{array}$ & $\begin{array}{l}\text { Support } \\
\text { person: Friend } \\
\text { or Family } \\
\end{array}$ & $\begin{array}{l}\text { Meeting/ } \\
\text { Coun- } \\
\text { seling }\end{array}$ & $\begin{array}{l}\text { Telep- } \\
\text { hone }\end{array}$ & $\begin{array}{l}\text { Nicotine } \\
\text { Replacement } \\
\text { Therapy } \\
\end{array}$ & Voucher & $\begin{array}{l}\text { Written } \\
\text { Material }\end{array}$ & $\begin{array}{l}\text { Audio/ } \\
\text { Video } \\
\text { Tapes }\end{array}$ & $\begin{array}{l}\text { Computer } \\
\text { Program }\end{array}$ & $\begin{array}{l}\text { Refer } \\
\text {-rals }\end{array}$ & $\begin{array}{l}\text { Home } \\
\text { Visit }\end{array}$ \\
\hline Albrect et al., 2006 & Yes & Yes & No & No & No & No & No & No & No & No \\
\hline Bryce et al., 2009 & Yes & Yes & Yes & No & No & No & No & No & No & No \\
\hline Bullock et al., 2009 & No & No & Yes & No & No & Yes & No & No & No & No \\
\hline $\begin{array}{l}\text { Campbell, et al., } \\
2006\end{array}$ & No & Yes & No & No & No & Yes & No & No & No & NO \\
\hline Cope et al., 2003 & No & Yes & No & No & No & Yes & No & No & No & No \\
\hline Dornelas et al., 2006 & No & Yes & Yes & No & No & No & No & No & No & No \\
\hline Edwards et al., 2008 & No & Yes & No & No & No & Yes & No & No & No & No \\
\hline $\begin{array}{l}\text { Ferreira-Borges, } \\
2005\end{array}$ & No & Yes & No & No & No & Yes & No & No & No & No \\
\hline Heil et al., 2008 & No & No & No & No & Yes & No & No & No & No & No \\
\hline $\begin{array}{l}\text { Hennrikus et al., } \\
2010\end{array}$ & Yes & Yes & Yes & No & No & No & Yes & No & No & No \\
\hline Jaakkola et al., 2001 & Yes & No & No & No & No & Yes & No & No & No & Yes \\
\hline Kataray et al., 2009 & No & Yes & No & No & No & Yes & No & No & No & Yes \\
\hline Lando et al., 2001 & No & No & Yes & No & No & Yes & No & No & No & No \\
\hline Lawrence et al., 2003 & No & Yes & No & No & No & Yes & No & Yes & No & No \\
\hline Malchodi et al., 2003 & No & Yes & Yes & No & No & No & No & No & No & Yes \\
\hline $\begin{array}{l}\text { McGowan et al., } \\
2010\end{array}$ & No & Yes & Yes & Yes & No & No & No & No & No & No \\
\hline McLeod et al., 2003 & No & Yes & No & No & No & No & No & No & No & No \\
\hline Moore et al., 2002 & No & No & No & No & No & Yes & No & No & No & No \\
\hline Ondersma et al. 2011 & No & Yes & No & No & Yes & Yes & No & Yes & No & No \\
\hline Patten et al., 2010 & No & No & Yes & No & No & Yes & Yes & No & No & No \\
\hline Peden et al., 2008 & No & Yes & No & No & No & No & Yes & No & No & No \\
\hline Vries et al., 2006 & Yes & No & No & No & No & Yes & Yes & No & No & No \\
\hline
\end{tabular}

The cognitive behavioral theory was utilized by two reviewed studies Albrect et al., (2006), and Peden et al. (2008) ${ }^{[18,19]}$. Cognitive behavioral theory is based on an individual's ability to learn new skills and apply learned rules to solve problems. "Cognitive behavioral strategies aim to decrease self-defeating behavior, such as smoking, by altering maladaptive perceptions through social support and therapeutic relationship, goal setting, reeducation, and urge control with an emphasis on teaching behavior control techniques” ${ }^{[18]}$. Diminishing negative thoughts, giving women tools to beat nicotine cravings, educating women on why a behavior change is necessary, and increasing self-efficacy could assist pregnant women in achieving abstinence from smoking ${ }^{[19]}$. Two studies used the social cognitive theory, including Patten et al. (2010) and Vries et al. (2006) ${ }^{[6,10]}$. This theory states that behaviors are learned through observation and personality, and represents a clinical approach to behavior change ${ }^{[21]}$. Bandura demonstrated the effects of the social cognitive theory by exposing children to a video showing aggressive and violent behaviors, and then placed the children in a room with a doll to see how they acted. The children who had seen the video reacted more violently and aggressively than the children who did not. The Patten et al. (2010) and Vries et al. (2006) studies used the social cognitive theory by distributing audio/video material to their participants ${ }^{[6,10]}$.

Vries (2006) and colleagues adopted the theory of planned behavior as part of the framework for the intervention ${ }^{[6]}$. The theory of planned behavior states that an individual's intentions are the most important factor in determining behavior. Ajzen's (2005) theory of planned behavior (TPB) is an extension of Ajzen and Fishbein's (1980) earlier theory of reasoned 
action (TRA) ${ }^{[22]}$. TPB provides a framework for understanding people’s behavior and its psychological determinants. Attitudes towards a behavior, subjective norms with respect to the behavior, and perceived control over the behavior are usually found to predict intentions and can serve as a weak point for attack in attempts to modify the behavior ${ }^{[22]}$. The level of belief must be examined in order to learn about the unique factors that influence one person to engage in a behavior and to prompt another to follow a different course of action ${ }^{[22]}$.

\subsection{I nitiation and duration of intervention}

The duration of each intervention varied significantly (see Table 1). The studies conducted by Hajek et al. (2001), Kataray et al. (2009), Malachodi et al. (2003), and Moore et al. (2002) included only those women who were in their first trimester of pregnancy ${ }^{[5,16,23,24]}$. Albrecht et al. (2006), Bullock et al. (2009), Dornelas et al. (2006), Ferreira-Borges (2005), Heil et al. (2008), Hennrikus et al. (2010), Ondersma et al. (2011), Patten et al. (2010), and Peden et al. (2008) allowed subjects to participate in the study up to and including the second trimester ${ }^{[3,18-20,25-29]}$. Eleven of the twenty-four studies either did not specify the time during the pregnancy for the initiation of the intervention or allowed subjects to join the study up until the last 12 weeks of gestation. There were no limitations on time of gestation for the studies conducted by multiple studies ${ }^{[2,6,17,30-37]}$.

There was a range in the studies based on the length of the intervention (see Table 1). Studies that implemented interventions on a short-term basis 1-3 months, included research performed by Bryce et al. (2009), Lando et al. (2001), Lawrence et al. (2003), Campbell et al. (2006), Edwards et al. (2008), Ferriera-Borges (2005), Hajek et al. (2001), McLeod et al. (2003) Ondersma et al. (2011) and Patten et al. (2010) ${ }^{[17,20,29-31,34,36]}$. Studies that used long term interventions or throughout the pregnancy included Albrecht et al. (2006), Bullock et al. (2009), Cope et al. (2003), Dornelas et al. (2006), Heil et al. (2008), Hennrikus et al. (2010), Jaakkola et al. (2001), McGowen et al. (2010), Moore et al. (2002), Oien et al. (2008), Peden et al. (2008), and Vries et al. (2006) ${ }^{[3,6,18,19,24,25,27,28,32,33,35,37]}$. Approximately half of the 24 studies followed the women after childbirth. However, the lengths of time varied greatly. Edwards et al. (2008) followed women for 1 week postpartum ${ }^{[2]}$; Lawrence et al. (2003) followed women for 10 days postpartum ${ }^{[17]}$; Vries et al. (2006) followed women for 6 weeks postpartum ${ }^{[6]}$; Ferriera-Borges (2005) followed women for 2 months postpartum ${ }^{[26]}$; Hennrikus et al. (2010) followed women for 3 months postpartum [28]; McLeod et al. (2003) followed women for 4 months postpartum [36]; Dornelas et al. (2006) and Hajek et al. (2001) followed women for 6 months postpartum ${ }^{[3,23]}$; Albrecht et al. (2006), Bryce et al. (2009), and Lando et al. (2001) followed women for 12 months postpartum ${ }^{[18,30,34]}$.

\subsection{Types of intervention}

Common interventions used included; meetings, telephone calls, written material, nicotine replacement, vouchers, audio and identification of a friend or a family member to assist in smoking cessation. The three most commonly used interventions were written material, meetings and telephone calls. Three studies used only one treatment modality, ten studies used two treatment modalities, and ten studies used three different modalities. Only one study used four treatment modalities.

\subsubsection{Meeting}

Fourteen of the Twenty-four studies reviewed in this paper included mainly implementing a regimen of meetings as part of their intervention in smoking cessation (see Table 2). The studies varied in their purpose of the meetings as well as duration and number of meetings. The study by Ferreira-Borges (2005) used one brief meeting with pregnant smokers for motivational interviewing, discussion of perceived barriers, and education about risks of smoking during pregnancy ${ }^{[26]}$. Motivational interviewing was a common theme between the studies that used meetings. Malchodi et al. (2003) conducted their intervention during 2 clinic visits and delivered smoking cessation counseling over 15 minutes from health care providers ${ }^{[5]}$. Role-playing was also used during the peer counseling meetings in this study. The study by Peden (2008) and colleagues aimed at reducing negative feelings and depressive symptoms by using self-affirmations and deep breathing during four 90-minute group meetings ${ }^{[19]}$. Hajek et al. (2001), McLeod et al. (2003), McGowan et al. (2010), Moore et al. (2002), and Oien et al. (2008) all used only one brief interventional meeting in combination with other interventional 
modalities ${ }^{[23,24,35-37]}$. Edwards et al. (2008) used 2 motivational interviews and Bryce et al. (2009) and Lawrence et al. (2003) both used three motivational interviews; all were varying in length ${ }^{[2,17,30]}$. Hennrikus et al. (2010), Katarary et al. (2009), and Vries et al. (2006) all used brief meetings at periodic times throughout the pregnancy ${ }^{[6,16,28]}$.

\subsubsection{Written material}

Thirteen of the Twenty-four studies reviewed included the distribution of written material to pregnant women as part of the intervention. Bullock et al. (2009) distributed literature to women that highlighted the importance of smoking cessation. The women received a total of 8 booklets in this study ${ }^{[25]}$. Campbell et al. (2009) mailed out information about smoking cessation to women in their study ${ }^{[31]}$. In the study by Cope, Nayyar, and Holder (2003), women were given handouts on smoking cessation at each clinic visit and were also given an invitation to come back to the clinic for more information on smoking cessation ${ }^{[32]}$. Edwards et al. (2009) distributed smoking quit guides with a commitment to quitting that the women signed, and telephone numbers for smoking cessation hotlines ${ }^{[2]}$. In Ferreira-Borges (2005) study, women were given a packet of information on risks of smoking during pregnancy, and benefits of quitting ${ }^{[26]}$. Booklets were distributed in the study by Hajek (2001) and colleagues, which discussed the risks of smoking and benefits of cessation, in addition to ways to stop smoking and remain abstinent. Quizzes were available at the end of each packet for the women to assess their knowledge ${ }^{[23]}$. Women were given materials to create a pregnancy scrapbook in the study by Hennrikus et al. (2010) ${ }^{[28]}$. Handouts with smoking cessation information were given to women and their partners in the study by Jaakkola, Zahlsen, and Jaakkola (2001) ${ }^{[33]}$. In Kataray et al. (2009) study, women were given brochures at the second home visit and were asked to keep a smoking diary during the third visit ${ }^{[16]}$. In the self-help study conducted by Moore et al. (2002), five different booklets were given to women (four through the mail) that focused on the pregnancy and the importance of smoking cessation. Literature was also available to friends and family ${ }^{[24]}$. In the study by Lawrence et al. (2003), six self-help manuals were given to women, one for each stage of change in the transtheoretical model and one for a friend. The self-help manuals consisted of an informational section followed by quizzes and exercises to promote the progression through the stages of change ${ }^{[17]}$. Patten et al. (2010) addressed common misconceptions and the cessation guide used was culturally appropriate for their Yupik population ${ }^{[20]}$. A self-help booklet called 'Stop Now for Your Baby' was given to women in the study by Lando et al. (2001) ${ }^{[34]}$. Booklets with smoking cessation information were given to the pregnant women and to their support person in the study by Vries et al. $(2006)^{[6]}$.

\subsubsection{Support person}

Six of the Twenty-four studies included a support person as part of the intervention. Women participating in the studies identified support persons as being a spouse, a significant other, another pregnant smoker, a friend or a family member. These interventions were diverse and included strategies such as creating a pregnancy scrap book, peer support meetings, and motivational interviewing ${ }^{[6,18,28,30,33,37]}$. These studies were based on the belief that smoking behaviors are influenced by the social system of the pregnant woman, and those closest to the pregnant smoker can positively influence the woman to quit smoking. Albrecht (2006) and colleagues determined that although a buddy system is effective in achieving short-term smoking cessation, long-term benefits may not exist ${ }^{[18]}$. Hennrikus et al. (2010) reported that female friends and family members who are not the pregnant woman's significant other may be quite influential, possibly more so than the woman's partner during smoking cessation efforts. They also discovered that friends might actually be more beneficial to smoking cessation efforts than family members ${ }^{[28]}$.

\subsubsection{Telephone contact}

Eight studies used a form of telephone contact with the participants. Dornelas et al. (2006) employed telephone contact bimonthly during pregnancy with the women to establish a link between psychological distress and the inability to quit smoking during pregnancy and then followed up monthly for 6 months postpartum by telephone ${ }^{[3]}$. McGowan et al. (2008) spoke with participants via telephone three times during the intervention and sent motivational text messages to participants ${ }^{[35]}$. Lando et al. (2001), Malchodi et al. (2003), and Patten et al. (2010) all used brief telephone interviews in combination with other modalities throughout the pregnancies ${ }^{[5,20,34]}$. Bryce et al. (2007) made only the initial contact with their participants via telephone. Bullock et al. (2009) made weekly interventional telephone contact with the 
participants ${ }^{[30]}$. Hennrikus et al. (2010) used monthly telephone interviews by midwives and other support persons as part of their smoking cessation intervention. Telephone follow-up calls were found not to be a feasible method of smoking cessation, because of the participants changing telephone numbers frequently, moved, or simply loosing contact, thus making it difficult to reach the participants after the counseling sessions ${ }^{[28]}$. Alternative methods of communications would be advised to pursue.

\subsubsection{Other interventions}

Ten studies used other treatment modalities, in addition to the four main modalities (meetings, written material, support persons, and telephone contact). Albrecht et al. (2006) used nicotine replacement therapy ${ }^{[18]}$. Audio/video tapes were distributed by Campbell et al. (2006), Patten et al. (2010), Peden et al. (2008), and Vries et al. (2006) ${ }^{[6,19,20,31]}$. Computer programs were issued by Campbell et al. (2006), Lawrence et al. (2003) and Ondersma et al. (2011) ${ }^{[17,29,31]}$. Hajek et al. (2001) added referrals for complimentary care to their smoking intervention plan ${ }^{[23]}$. Home visits were a part of Jaakkola et al. (2001), Kataray et al. (2009), and Malchodi et al. (2003) studies ${ }^{[5,16,33]}$.

\section{Discussion}

Approximately half of the studies did not specify a time during pregnancy when the intervention started. Intervention mentioned above occurred during pregnancy. According to McLeod (2004) and colleagues the period of time in early pregnancy is peak opportunity for education, specifically about smoking cessation ${ }^{[36]}$. It would be highly beneficial if the interventions last throughout the women's pregnancy to ensure smoking cessation through the duration of the post partum period. The majority of the studies followed the participants during postpartum, varying from 1 week to 12 months. Bryce et al. (2006) found that at a 12-month follow up $16.5 \%$ participants remained smoke free ${ }^{[30]}$. The Community Action of Tobacco for Children's Health (CATCH) study which consisted of regular contact, personal ongoing support and motivation were viewed as the main contributing factors in enhancing confidence to tackle smoking ${ }^{[26]}$. Continuation of this support and encouragement beyond a successful quit attempt were important in maintaining the non-smoking status in this study.

Only three studies used one treatment in their intervention modality. Heil et al. (2008) used only vouchers ${ }^{[27]}$. McLeod et al. (2003) used only meetings, and Moore et al. (2002) used only written materials ${ }^{[24,36]}$. The majority of the studies included multiple modalities in their smoking cessation interventions, 11 of the 24 studies employed three or more. Each intervention was significantly different in terms of intensity of the intervention, gestational age, and person implementing the intervention, so comparing the interventions side by side is difficult. It is unclear at this point whether the presence of multiple treatment modalities leads to greater rates of smoking cessation during pregnancy.

The findings from this integrative review emphasize the difficulties associated with developing health services for women who smoke during pregnancy. However, most of the studies resulted in smoking cessation or reduction. Fifteen out of the 24 reviewed studies showed either a decrease in cigarette use or smoking cessation completely. Eight articles showed no change, and no study showed an increase in amount of cigarettes smoked. One study in particular showed greater smoking cessation rates in the control group than the experimental group ${ }^{[20]}$. Table 1 discusses the outcomes of each study in detail. Overall, the interventions were successful at achieving smoking cessation or a reduction in smoking.

There was inconsistent evidence regarding results obtained from each intervention implemented. For example, FerreiraBorges (2005) and Hajek et al. (2001) both implemented an intervention that consisted of meetings and/or counseling and written material ${ }^{[23,26]}$. However Ferreira-Borges (2005) achieved a significant reduction in cigarette smoking while Hajek et al. (2001) did not see any influence from the interventions ${ }^{[26]}$. Hejak et al. (2001) reported that the lack of recruitment and implementation of smoking cessation during the study corresponded with the lack of midwifery knowledge and involvement within the study ${ }^{[23]}$. However, Ferreira-Borges (2005) obtained a higher success rate of smoking cessation due to the person to person contact, session length, total amount of contact time, various types of clinicians and 
counseling ${ }^{[26]}$. Continuous ongoing support and motivation were the main contributing intervention factors. CATCH intervention by Ferreira-Borges also used a 12-month follow up by the external evaluation team. Using a standard intervention for all types of pregnant women may not be effective as the complexities of pregnancy itself contribute to the variability between women and magnify the need for individual interventions. For example, the study by Albrecht and Colleagues (2006) focused on only adolescent pregnant women. The study offered an initial attempt to intervene with pregnant adolescent smokers in a randomized controlled trial. This study demonstrated that a developmentally appropriate cognitive-behavioral smoking cessation program with peer support was effective in short-term cessation of smoking among pregnant adolescents. Findings confirmed the importance of peer support in the modification of pregnant adolescent smoking behavior ${ }^{[18]}$.

Community outreach workers, public health nurses, general practitioners, and midwives were used to conduct the intervention in different studies. Some studies reported providing training to the practitioners while others did not mandate it. The variability in the preparation of the person conducting this intervention may have contributed to the variability in the results. For example, Vries et al. (2006) reported favorable outcomes when interventions are implemented by specifically trained health professionals ${ }^{[6]}$.

Continued efforts to reduce and eliminate smoking during pregnancy are an essential component of a plan to drastically improve maternal and infant health. After evaluating studies included in this review, it is clear that more data needs to be gathered about age appropriate smoking cessation programs, training of professionals conducting the interventions, duration of appropriate smoking cessation programs and introducing smoking cessation before pregnancy occurs. There is also a need for collecting qualitative data from participating pregnant women to be able increase the efficacy of interventions trialed and allow interventions to be tailored to women's specific needs. The inconsistent data gathered from reviewed studies should inform health care providers that the population of women who smoke during pregnancy cannot be lumped into a single category but must be thoroughly explored so their diverse backgrounds, beliefs, and needs are properly accounted for.

This integrative literature review confirmed that pregnant smokers are willing to take action and assume responsibility for the health and well-being of themselves and their unborn babies and will utilize resources when available to achieve smoking cessation. Smoking cessation interventions need to be user friendly, flexible, accessible, culturally sensitive, age appropriate and effective. Increasing the knowledge of pregnant smokers is an integral part of a successful intervention. Of the reviewed studies, most achieved some decrease in smoking. A decrease in smoking is beneficial to mother and baby and a worthwhile goal of smoking cessation interventions.

\subsection{Study limitations}

A potential limitation of this review is that the literature search was limited to articles and journals retrieved only from the CINAHL and MEDLINE search engines and through the ancestory approach. This method may increase the likelihood of inadequate sampling. Other articles undoubtedly exist, but were eliminated from this review if they were not found on the search engine at the time of review, possible creating a bias in the study. Utilizing multiple methods for obtaining research articles is essential for increasing validity of the integrative review ${ }^{[13]}$.

While most of the smoking cessation interventions, in the reviewed studies, proved to be beneficial either at smoking cessation or smoking reduction, most of them had limitations. The most common limitation was variability in the sample size ranging from 16 to 12,133. Another limitation was in regard to the persons conducting the intervention and their level of training. Some studies used nurse midwives, others used community outreach workers, and others used various volunteers.

\subsection{Recommendations}

Biochemically confirmed smoking status should be one of the smoking cessation programs'outcome measures, and the program should begin at the beginning of pregnancy. This belief is supported by the involvement of a pregnant woman's 
family members, friends, or partner may to positively influencing smoking abstinence. It is clear that due to significant variability among pregnant women, each intervention will need to have aspects specifically geared toward the targeted population. An intervention with a strong theoretical basis with reproducible components can and should be at the foundation of smoking cessation programs for pregnant women.

\section{Conclusion}

The objective of this integrative review was review the varying interventions used to promote the cessation of smoking in pregnant women, and to present the current state of knowledge pertaining to the use and efficacy of the various intervenetions employed. There was inconsistent data obtained from the studies included in this review in terms of the results yielded from each treatment modality. The samples, interventions, and measurement points contributed to the inconsistency in their results. However, the majority of the studies included in this review supported their interventions with either biochemically confirmed smoking cessation or smoking reduction.

Because of limitations and inconsistency in findings, additional research is needed to determine the exact effect of each intervention and its effect on the smoking status of pregnant women. Experimental and qualitative studies are needed to further investigate the differences between interventions such as written materials, identification of a support person, telephone contact, and counseling meetings when implemented individually in various populations. Additionally, further research is needed to evaluate individual treatment modalities using a side-by-side comparison.

\section{References}

[1] McClure J. US National Library of Medicine National Institutes of Health. Apr 2004; 6 Suppl 2:S153-61Motivating prepartum smoking cessation: a consideration of biomarker feedback. Available from: http://www.ncbi.nlm.nih.gov/pubmed/15203818

[2] Edwards, M. J., Geiser, T., Chafin, C., Weatherby, N. L., \& Smith, C. S.M.A.R.T. mothers are resisting tobacco: Prenatal smoking cessation in WIC mothers. Journal of Allied Heatlh. 2009; 38(3): 170-176. Available from: MEDLINE database PMid:19753429

[3] Dornelas, E. A., Magnavita, J., Beazoglou, T., Fischer, E. H., Oncken, C., Lando, H., et al. Efficacy and cost-effectiveness of a clinic-based counseling intervention tested in an ethnically diverse sample of pregnant smokers. Patient Education and Counseling. 2006; 64(1-3): 342-349. PMid:16859864 http://dx.doi.org/10.1016/j.pec.2006.03.015

[4] Peterson, Z., Steyn, K., Everett-Murphy, K., \& Emmelin, M. Pregnant women’s responses to a tailored smoking cessation intervention: Turning hopelessness into competence. Global Health Action. 2010; 14(3): 1-9.

[5] Martiz, G. S., \& Harding, S. Life-long programming implications of exposure to tobacco and nicotine before and soon after birth: Evidence for altered lung development. International Journal of Environmental Research and Public Health. 2011; 8(3): 875-898. PMid:21556184 http://dx.doi.org/10.3390/ijerph8030875

[6] Centers for Disease Control and Prevention (2004). Available from: http://www.cdc.gov/tobacco/data_statistics/sgr/2004/highlights/reproductive/index.htm

[7] Cooper, H. M. The Integrative research review: A systematic approach. Beverly Hills: Sage; 1984.

[8] Cooper, H. M. Synthesizing research: A guide for literature reviews (3rd ed.). Thousand Oaks: Sage; 1998.

[9] Malchodi, J. B., Filho, P. V., Peterson, G. O., \& Chatkin, J. M. Quantitative effects of tobacco smoking exposure on the maternal-fetal circulation. Biomed Central. 2011; 11(24): 1-6.

[10] Whittemore, R., \& Knafl, K. The integrative review: Updated methodology. Journal of Advanced Nursing. 2005; 52(5): 546-553. PMid:16268861 http://dx.doi.org/10.1111/j.1365-2648.2005.03621.x

[11] Cooper, H. M. Scientific guidelines for conducting integrative research reviews. Review of Educational Research. 1982; 52: 291-302. http://dx.doi.org/10.3102/00346543052002291

[12] Crabtree, B. F., \& Miller, W. L. Doing qualitative research (2nd ed.). Newbury, CA: Sage; 1992.

[13] Stetler, C. B., Morsi, D., Rucki, S., Broughton, S., Corrigan, B., Fitzgerald, J., et al. Utilization-focused integrative reviews in a nursing service. Applied Nursing Research. 1998; 11: 195-206. http://dx.doi.org/10.1016/S0897-1897(98)80329-7

[14] Kataray, G., Kublay, G., \& Emiroglu, O. N. Effect of motivational interviewing on smoking cessation in pregnant women. Journal of Advanced Nursing. 2010; 66(6): 1328-1337. http://dx.doi.org/10.1111/j.1365-2648.2010.05267.x

[15] Lawrence, T., Aveyard, P., Evans, O., \& Cheng, K. K. A cluster randomized controlled trial of smoking cessation in pregnant women comparing interventions based on the transtheoretical (stages of change) model to standard care. Tobacco Control. 2003; 12(2): 168-177. PMid:12773727 http://dx.doi.org/10.1136/tc.12.2.168

Published by Sciedu Press 
[16] Albrecht, S. A., Caruthers, D., Patrick, T., Reynolds, M., Salamie, D., Higgins, L. W., et al. A randomized controlled trial of a smoking cessation intervention for pregnant adolescents. Nursing Research. 2006; 55(6): 402-410. PMid:17133147 http://dx.doi.org/10.1097/00006199-200611000-00004

[17] Peden, A. R., Rayens, M. K., Hall, L. A., Hahn, E., Riker, C., Ashford, K., et al. Nicotine addiction in pregnancy: Preliminary efficacy of a mental health intervention. Addictive Disorders \& Their Treatment. 2008; 7(4): 179-189. http://dx.doi.org/10.1097/ADT.0b013e3181484768

[18] Vries, H., Bakker, M., Mullen, P. D., \& Van Breukelen, G. The effects of smoking cessation counseling by midwives on dutch pregnant women and their partners. Patient Education and Counseling. 2005; 63(1-2): 177-187. PMid:16406475 http://dx.doi.org/10.1016/j.pec.2005.10.002

[19] Bandura, A. Health Promotion by social cognitive means. Health Education \& Behavior. 2004; 31: 143-164. PMid:15090118 http://dx.doi.org/10.1177/1090198104263660

[20] Ajzen. Attitudes, Personality, and Behavior (2nd edition). Milton-Keynes, England: Open University Press / McGraw- Hill; 2005.

[21] Bullock, L., Everett, K. D., Dolan Mullen, P., Geden, E., Longo, D. R., \& Madsen, R. Baby BEEP: A randomized controlled trial of nurses' individualized social support for poor rural pregnant smokers. Maternal \& Child Health Journal. 2009; 13(3): 395-406. PMid:18496746 http://dx.doi.org/10.1007/s10995-008-0363-z

[22] Ferreira-Borges, C. Effectiveness of a brief counseling and behavioral intervention for smoking cessation in pregnant women. Preventive Medicine. 2005; 41(1): 295-302. PMid:15917025 http://dx.doi.org/10.1016/j.ypmed.2004.11.013

[23] Heil, S. H., Stephen, H. T., Bernstein, I. M., Solomon, L. J., Rogers, R. E., Thomas, C. S., et al. Effects of voucher-based incentives on abstinence from cigarette smoking and fetal growth among pregnant women. Addiction. 2008; 103(6): 1009-1018. PMid:18482424 http://dx.doi.org/10.1111/j.1360-0443.2008.02237.x

[24] Hennrikus, D., Pirie, P., Hellerstedt, W., Lando, H. A., Steele, J., \& Dunn, C. Increasing support for smoking cessation during pregnancy and postpartum: Results of a randomized controlled pilot study. Preventive Medicine. 2010; 50(3): $134-137$. PMid:20079760 http://dx.doi.org/10.1016/j.ypmed.2010.01.003

[25] Ondersma, S.J., Svikis, D. S., Lam, P.K., Connors-Burge, V.S., Ledgerwood, D.M., \& Hopper, J.A. A randomized trial of computer-delivered brief intervention and low-intensity contingency management for smoking during pregnancy. Nicotine \& tobacco research. 2011; (14)3: 351-360.

[26] Patten, C. A., Windsor, R. A., Renner, C. C., Enoch, C., Hochreiter, A., Nevak, C., et al. Feasibility of a tobacco cessation intervention for pregnant Alaska native women. Nicotine \& Tobacco Research. 2010; 12(2): 79-87. PMid:20018946 http://dx.doi.org/10.1093/ntr/ntp180

[27] Bryce, A., Butler, C., Gnich, W., Sheehy, C., \& Tappin, D. CATCH: development of a home-based midwifery intervention to support young pregnant smokers to quit. Midwifery. 2009; 25(5): 473-482. PMid:18280015 http://dx.doi.org/10.1016/j.midw.2007.10.006

[28] Campbell, E., Walsh, R., Sanson-Fisher, R., Burrows, S., \& Stojanovski, E. A group randomised trial of two methods for disseminating a smoking cessation programme to public antenatal clinics: effects on patient outcomes. Tobacco Control. 2006; 15(20): 97-102. PMid:16565456 http://dx.doi.org/10.1136/tc.2004.011049

[29] Cope, G. F., Nayyar, P., \& Holder, R. Feedback from a point-of-care test for nicotine intake to reduce smoking during pregnancy. The Association of Clinical Biochemists. 2003; 40(6): 674-679.

[30] Jaakkola, N., Zahlsen, K., \& Jaakkola, J. K. Effects of a population-based smoking cessation programme on smoking in pregnancy. European Journal of Public Health. 2001; 11(4): 446-449. PMid:11766488 http://dx.doi.org/10.1093/eurpub/11.4.446

[31] Lando, H. A., Valanis, B. G., Lichtenstein, E., Curry, S. J., McBride, C. M., Pirie, P. L., et al. Promoting smoking abstinence in pregnant and postpartum patients: A comparison of 2 approaches. The American Journal of Managed Care. 2001; 7(7): 685-693. PMid:11464427

[32] McGowan, A., Hamilton, S., Barnett, D., Nsofor, M., Proudfoot, J., \& Tappin, D. 'Breathe': the stop smoking service for pregnant women in Glasgow. Midwifery. 2010; 26(3): 1-13. PMid:18692285 http://dx.doi.org/10.1016/j.midw.2008.05.005

[33] McLeod, D, Pullon, S, Benn, C, Cookson, T, Dowell, A, Viccars, A, et al. Can support and education for smoking cessation and reduction be provided effectively by midwives within primary maternity care? Midwifery. 2013; 20(10): 37-50.

[34] Oien, T., Storro, O., Jenssen, J. A., Johnsen, R. The impact of a minimal smoking cessation intervention for pregnant women and their partners on a perinatal smoking behavior in primary health care: A real-life controlled study. BMC Public Health. 2008; 8: 325-335. PMid:18808705 http://dx.doi.org/10.1186/1471-2458-8-325

[35] Hajek, P., West, R., Lee, A., Foulds, J., Owen, L., Eiser, J. R., et al. Randomized controlled trial of a midwife-delivered brief smoking cessation intervention in pregnancy. Addiction. 2001; 96(3): 485-494. PMid:11255587 http://dx.doi.org/10.1046/j.1360-0443.2001.96348511.x

[36] Moore, L., Campbell, R., Whelan, A., Mills, N., Lupton, P., Misselbrook, E., et al. Self help smoking cessation in pregnancy: cluster randomized controlled trial. BMJ. 2002; 325(7377): 1383. PMid:12480850 http://dx.doi.org/10.1136/bmj.325.7377.1383 